\title{
HUNTING FOR GLOBULAR CLUSTER CANDIDATES IN THE FAR HALO (d > 20 kpc) OF M 31 WITH THE TAUTENBURG SCHMIDT TELESCOPE
}

\author{
L. FEDERICI ${ }^{1}$, F. BONOLI ${ }^{2}$, F. FUSI PECCI ${ }^{1}$, B. MARANO ${ }^{1}$, F. BÖRNGEN ${ }^{3}$ and \\ H. MEUSINGER ${ }^{3}$ \\ ${ }^{1}$ Osservatorio Astronomico, Bologna, Italy \\ ${ }^{2}$ Dipartmento di Astronomia, Bologna, Italy \\ ${ }^{3}$ Karl-Schwarzschild-Observatorium, Tautenburg, Germany
}

\section{Introduction}

The M 31 cluster system is the largest sample of globular clusters found in the Local Group, is sufficiently close to allow quite easy observations, and is negligibly affected by total reddening, at least for a large outer sub-set. Unfortunately, a complete and uncontaminated sample of M 31 clusters is not available yet for two basic reasons:

1) since at the $M 31$ distance the cluster sizes are comparable with the seeing-disk (10 pc $\sim 3.3$ arcsec), selections made via morphological-visual inspection of candidates (see for references Federici et al. 1993) fails to select the most compact clusters in the inner areas (mistaken for stars), and the most extended ones in the outer halo (mistaken for background galaxies);

2) the far halo of $M 31$, outside $20 \mathrm{kpc}$, has never been surveyed with deep images taken in sufficiently good seeing conditions and with large-scale plates or CCD-frames.

On the other hand, since to cover properly the M 31 halo (i.e. $100 \mathrm{kpc}$ ) it is necessary to survey an area of about $9 \times 9 \mathrm{deg}^{2}$, it is almost impossible to identify candidates using straight exceedingly high-quality, high-resolution, small field imaging. Therefore, surveys based on 'classic' wide-field imaging are still the most efficient way to select G.C. candidates.

As stressed by van den Bergh (1964) and proven by our early survey (Battistini et al. 1987), the 2-m Schmidt telescope at the Karl-Schwarzschild Observatorium in Tautenburg is the most suitable for this kind of search since it offers a wide field coupled with a still sufficient platescale ( $3 \times 3 \mathrm{deg}, 51.6 \mathrm{arcsec} / \mathrm{mm}$ ). In particular, though this observational set-up excludes the possibility of selecting 'compact' candidates, it will allow the detection of some bright clusters similar to NGC 2419 in the Milky Way, located at about $100 \mathrm{kpc}$ from the Galactic nucleus and with $M_{v} \sim-9.5$ (corresponding to $V \sim 15$ at the $M 31$ distance). If discovered in $M 31$, such far clusters would be crucial tools for studying chemical evolution and dynamics of its halo.

To this purpose a number of BVI plates have been obtained with the Tautenburg Schmidt telescope, covering the entire body of $M 31$ up to a radius of about $70 \mathrm{kpc}$ (see the composite global map). They will be inspected to select GC-candidates, following the criteria adopted in our previous surveys and briefly outlined below. 


\section{Previous Searches for GCs in M 31: the 'Current Best Sample'}

All the major selections have been carried out by eye inspection on large scale plates and by morphological classification of the images. A first de-contamination from spurious objects was usually made by cross-checking with lists of known foreground and background objects, HII regions, PNs etc. The total sample of candidates inserted at least in one catalogue includes so far 1028 objects ranging from $\mathrm{V} \sim 14$ down to $\sim 19.5$ and spanning a radial distance from 0 to $\sim 30 \mathrm{Kpc}$. However, the number of candidates in common in the three latest major catalogues (Sargent et al. 1977; Crampton et al. 1985; Battistini et al. 1980, 1987, 1992) is 200, and they constitute the bulk of the confirmed M 31 clusters.

Contamination: Most of the spurious sources are foreground stars, background galaxies, and some peculiar objects (e.g. HII regions and PN). Their relative impact on the samples varies quite strongly with varying galactocentric distance and orientation with respect to the $M 31$ main axes. Three basic techniques have been used to confirm or exclude the intrinsic cluster nature of the candidates: radial velocities, 181 clusters confirmed so far (Huchra et al. 1991; Federici et al. 1990, 1992); CCD high-resolution imaging, 81 globulars (48 with known radial velocities) (Racine, 1991; Racine \& Harris 1992; Cohen \& Freeman 1991); integrated colour indices and image structure (Reed et al. 1992). Since in the outer uncontaminated regions of $M 31$ almost none of the cluster confirmed via the two previous techniques have $B-V>1.0$, red objects located or projected outside the main body of $M 31$ can be rejected, but unfortunately candidates with 'appropriate' colours can well be galaxies (see Reed et al. 1992 and Battistini et al. 1980, 1987, 1992, for a discussion).

At present 214 clusters have been confirmed with at least one reliable technique, including a few probable members to NGC 205.

Completeness: We believe that as a result of the surveys published to date, the search can be considered to be fairly complete in the surveyed areas to $\mathrm{V} \sim 18\left(\mathrm{M}_{\mathrm{V}} \sim-6.5\right)$. However, completeness can hardly be claimed even at bright magnitudes for very compact clusters (particularly when superimposed on a strong background) or very loose objects in the outer halo.

In the latest study carried out by our group (Battistini et al. 1993, hereafter Bo93), we have defined the current best sample of M 31 GCs as the sample which includes:

- outside isophote No. 1 (de Vaucouleurs 1958);

a) all the candidates confirmed to be clusters via high-resolution imaging or spectroscopy (71 objects);

b) all the candidates with (B - V) $\leq 1.10$ even if not confirmed via the quoted techniques (2 objects in total).

- inside isophote No. 1;

c) all the candidates confirmed to be clusters via high-resolution imaging or spectroscopy (138 objects);

d) all the candidates even if not confirmed via the quoted techniques (152 objects in total), but excluding from this number 7 candidates still lacking reliable colours, and 15 clusters having $(B-V)>1.1$ and superimposed on a very low background (galaxies?).

In conclusion, the total number of objects included in the 'current best sample' is 341 . Out of these, 81 have been confirmed via high resolution imaging, 176 via spectroscopy, 48 via both methods. Only 6 G.C. of the 'c.b.s' lie at a projected distance larger than $20 \mathrm{kpc}$. 


\section{Expected Number of GCs in the Outer Halo of M 31}

A reliable estimate of the total number of $\mathrm{M} 31 \mathrm{GCs}$ is affected by the following sources of error:

1) residual contamination in the adopted sample;

2) incompleteness for very compact and/or very faint objects; and

3) lack of data for the residual unsurveyed areas of $M 31$.

The correct quantitative evaluation of these aspects is very difficult, and Racine (1991) and Bo93 have recently tackled the problem. Their conclusions concerning the ratio between the $M 31$ and Milky Way GC populations are different (though compatible within the errors), leading to $N(M 31) / N(M W) \sim 2$ or 3, respectively. Based on our quoted analysis, we believe that the total number of $M 31 \mathrm{GCs}$ should be $\sim 400_{-40}^{+70}$, where the error bar has been assigned just on the basis of the experience made by working for years on the candidates.

Assuming for example that the cluster population ratio between the two galaxies is 2.5 , and considering that outside $20 \mathrm{kpc}$ in the Milky Way there are 12 clusters brighter and 13 fainter than $M_{V}=-6.5$, one could expect to find about 30 clusters brighter and 33 fainter than $V=18$ in $M 31$.

If one then restricts the comparison to the region between 20 and $30 \mathrm{kpc}$, already partially reached by the available surveys, there are 9 clusters ( 4 of which brighter are than $M_{v}=-6.5$ ) in our own galaxy, hence, adopting the ratio of 2.5 , about 23 clusters are expected in $M 31,10$ of which are brighter than $V=18$. Since 6 bright clusters have already been discovered in this area, one can expect to find $15-20$ more clusters, mostly fainter than $V=18$. Due to the low surface brightness expected for clusters at these galactocentric distances (Palomar-like objects are found in the Milky Way) and the large area to be scanned ( 12 sq. deg.), the task of finding a few other clusters appears very hard: at $\mathrm{V} \sim 19.5$, for each square degree one has to check many thousands of images in order to pick up about 1 globular cluster together with many misclassified galaxies, morphologically and photometrically identical to globular clusters.

The game of detecting fainter candidates within $30 \mathrm{kpc}$ or bright clusters in the outermost halo is even more challenging. The number of images to be inspected steeply increases with magnitude as does the area to survey going farther and farther away. Moreover, the incidence of sparse Palomar-like objects probably increases in M 31 too.

\section{References}

Auriere, M., Coupinot, G. and Hecquer, J., 1992. Astron. Astrophys., 256, 95.

Battistini, P.L., Bonoli, R., Braccesi, A., Fusi Pecci, F., Malagnini, M.L. and Marano, B., 1980. Astron. Astrophys. Suppl., 42, 357.

Battistini, P.L., Bonoli, R., Braccesi, A., Federici, L., Fusi Pecci, F., Marano, B. and Börngen, F., 1987. Astron. Astrophys. Suppl., 67, 447.

Battistini, P.L., Bonoli, F., Casavecchia, M., Ciotti, L., Federici, L. and Fusi Pecci, F., 1993. Astron. Astrophys., 272, 77.

Cohen, J.G. and Freeman, K.C., 1991. Astron. J., 101, 483.

Crampton, D., Cowley, A.P., Schade, D. and Chayer, P., 1985. Astrophys. J., 288, 494.

Federici, L., Fusi Pecci, F and Marano, B., 1990. Astron. Astrophys., 236, 99.

Federici, L., Bonoli, F., Ciotti, L., Fusi Pecci, F., Marano, B., Lipovetsky, V.A., Neizvestny, S.I. and Spassova, N., 1993. Astron. Astrophys., 274, 87. 
Huchra, J.P., Brodie, J.P. and Kent, S.M., 1991. Astrophys. J., 370, 495.

Racine, R., 1991. Astron. J., 101, 865.

Racine, R. and Harris, W.E., 1992. Astron. J., 104, 1068.

Reed, L.G., Harris, G.L.H. and Harris, W.E., 1992. Astron. J., 103, 824.

Sargent, W.L.W., Kowal, S.T., Hartwick, F.D.A. and van den Bergh, S., 1977. Astron. J., 82, 947.

de Vaucouleurs, G., 1958. Astrophys. J., 128, 465.

van den Bergh, S., 1969. Astrophys. J. Suppl., 19, 145. 